\title{
Estimating Correspondences of Deformable Objects "In-the-wild"
}

\author{
Yuxiang Zhou ${ }^{\star}$ Epameinondas Antonakos ${ }^{\star} \quad$ Joan Alabort-i-Medina ${ }^{\star} \quad$ Anastasios Roussos \\ Stefanos Zafeiriou ${ }^{\star}, \dagger$ \\ ${ }^{\star}$ Department of Computing, Imperial College London, U.K. \\ ${ }^{\dagger}$ Center for Machine Vision and Signal Analysis, University of Oulu, Finland \\ \{yuxiang.zhou10, e.antonakos, ja310, troussos, s.zafeiriou\}@imperial.ac.uk
}

\begin{abstract}
During the past few years we have witnessed the development of many methodologies for building and fitting Statistical Deformable Models (SDMs). The construction of accurate SDMs requires careful annotation of images with regards to a consistent set of landmarks. However, the manual annotation of a large amount of images is a tedious, laborious and expensive procedure. Furthermore, for several deformable objects, e.g. human body, it is difficult to define a consistent set of landmarks, and, thus, it becomes impossible to train humans in order to accurately annotate a collection of images. Nevertheless, for the majority of objects, it is possible to extract the shape by object segmentation or even by shape drawing. In this paper, we show for the first time, to the best of our knowledge, that it is possible to construct SDMs by putting object shapes in dense correspondence. Such SDMs can be built with much less effort for a large battery of objects. Additionally, we show that, by sampling the dense model, a part-based SDM can be learned with its parts being in correspondence. We employ our framework to develop SDMs of human arms and legs, which can be used for the segmentation of the outline of the human body, as well as to provide better and more consistent annotations for body joints.
\end{abstract}

\section{Introduction}

Statistical Deformable Models (SDMs) of various objects is a well-studied and popular area in the intersection of computer vision and machine learning [26, 24, 43, 54, 14, 64, 60]. Recently, we have witnessed tremendous developments on SDMs of human faces and bodies trained with images that are captured under unconstrained conditions, usually referred to as "in-the-wild" $[14,21,64,60$, $11,59,12,35,2,63,8,9,3,57]$. This is attributed to:

- The abundance of complex visual data, spread through web services (e.g. Youtube, Flickr, Google Images),

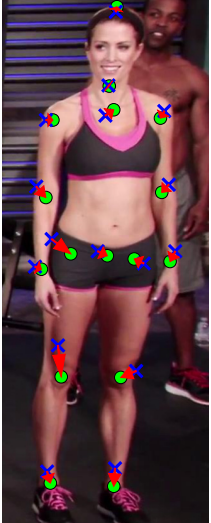

(a) MPII

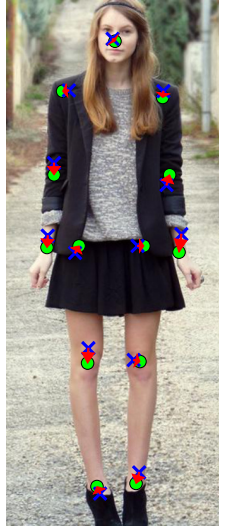

(b) Fashion

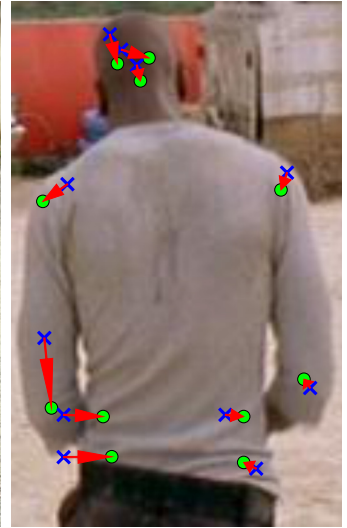

(c) FLIC
Figure 1: Examples of inconsistent annotations of human pose among different datasets. Blue markers denote the original annotations. The arrows and green markers show the correct location at which the points should be annotated.

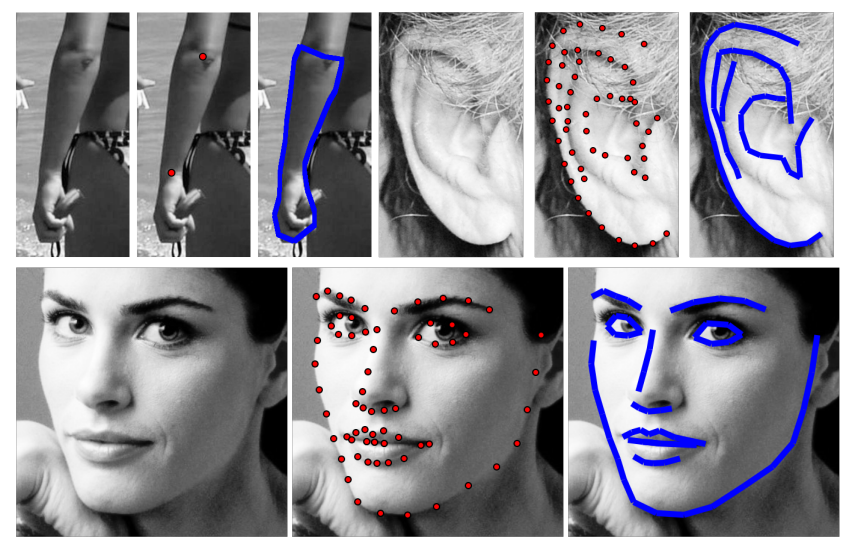

Figure 2: Comparison of the standard landmark annotation (red dots) with the curve annotation (blue lines) on arms, ears and faces. It is evident that the curve annotations surpass the inevitable inconsistency of sparse annotations.

which has led to the development of "in-the-wild" databases of human faces and bodies [14, 37, 64, 20]. 
- The manual annotation of such databases that has been undertaken by several research teams $[51,22,29,7]$.

- The development of powerful visual features that are able to describe objects and their parts in a robust manner (e.g., SIFT [41], HoGs [27] ), as well as generative and discriminative methodologies for learning SDMs.

However, there are two main drawbacks when building SDMs directly on manually annotated landmarks:

- Annotating with regards to consistent landmarks is an extremely time-consuming, tedious and labour intensive work [52], which is usually performed by a trained person. Furthermore, for various object classes, it requires a highly skilled person in order to identify and annotate landmarks in a consistent manner. For example, the human ear has very complicated inner structures (helix, crus antihelicis, scapha, tragus, lobe etc.) which remarkably differ between different ears. Moreover, certain ear parts, such as fossa triangularies and crus helicis, do not appear in all ears and their visibility is highly sensitive to the head pose and illumination variation. Another such example is the human body, which is generally annotated with regards to a number of landmarks that intuitively correspond to a set of body joints. For most body pose databases, the annotation task was undertaken by a crowd-sourcing Internet marketplace, so-called Amazon Mechanical Turk (AMT). Unfortunately, this resulted in acquiring inconsistent and inaccurate annotations, in many cases ${ }^{1}$ (please see Figure 1). As it was also recently pointed out [56], the inconsistencies in body joint annotations may also render the comparison between different human pose estimation methodologies irrelevant.

- The nature of many deformable objects does not allow them to be annotated with regards to a consistent set of landmarks (e.g., bottles, fruits etc.). Additionally, it is very difficult to consistently annotate the outline of certain objects such as faces, ears, body, since these landmarks do not have any semantic meaning. That is why many state-of-the-art methods opt to leave the boundary out when reporting results [59, 12]. The majority of the state-of-the-art methods for model-based landmark localisation [21, 64, 60, 59, 12] are not applicable to objects with inconsistent sets of landmarks.

To illustrate how time-consuming careful annotation of a complex deformable object is, we lay down our own experience based on the human ear. A trained annotator needs an average of 4 minutes per image for the manual annotation

\footnotetext{
${ }^{1}$ In the case of faces, the quality of annotations produced from AMT are extremely inaccurate and cannot, by any means, be compared with the ones provided by the recent $300 \mathrm{~W}$ competition $[52,51]$.
}

of 55 landmarks. This means that the annotation of 1000 images requires a total of about 67 hours. Furthermore, the quality of training as well as fatigue greatly influence the annotation accuracy. Hence, a second pass on the annotated data is, in many cases, necessary. Due to the fact that manual annotation is a costly and labour-intensive procedure, unsupervised learning of deformable models for the task of object alignment has recently attracted some attention [30, 13, 25, 34, 32, 36, 33, 40, 62]. However, because the problem of fully unsupervised discovery of the deformations of arbitrary objects is difficult and ill-posed, the limited number of methods that have been proposed for the task cannot be directly applied to arbitrary collections of "in-thewild" images. On the other hand, the method of [10], which can deal with "in-the-wild" images, requires a set of consistently annotated sparse shapes to perform deformable face congealing.

\section{Contributions}

In this paper, we propose a solution for annotating an object with regards to its deformations that requires considerably less effort compared to manual annotation and, at the same time, can be used to define statistical deformations for objects without a consistent set of landmarks. We employ the proposed method in order to construct SDMs based on the outline of human body parts (i.e., arms and legs). The proposed SDM can also be used to provide accurate and consistent annotations for several of the body joints (such as wrist, elbow etc.). To this end, we argue and empirically demonstrate that it is better to annotate an object with regards to a set of continuous lines that describe its shape. An example is provided in Figure 2, which compares the standard landmark annotations that are employed in the current literature with the proposed curve annotations for arms, ears and faces. It becomes evident that the curve annotations avoid the inherent ambiguity of placing sparse landmarks and offer a richer description of the object's shape. Furthermore, these curves can be automatically generated by recently proposed methods that perform discriminative segmentation of objects [42,39]. Note that the work in [65] is the only one that shows that training SDMs based on the outline contours of the human body parts has considerable advantages compared to using the sparse skeleton joints, as done by the majority of existing SDMs for human pose.

Furthermore, we capitalise on recent advances on multiframe optical flow estimation [31, 49, 55] and show that the relevant methodologies have matured enough to densely annotate the proposed shapes using either simplistic or even more sophisticated and robust shape representation methods [44]. In particular, in order to build dense correspondences between different shape instances of the same object class, we jointly estimate the optical flow among all the instances by imposing low-rank constrains, an approach that we call 
Shape Flow. Multiframe optical flow has originally been applied on video sequences, relying on the assumptions of colour consistency and motion smoothness [31]. However, these assumptions do not hold in our case, where we have a collection of shapes. Therefore, we introduce appropriate modifications based on the consistency of image-based shape representation, as well as low-rank priors.

Additionally, we show that the proposed methodology can be applied on landmark localisation, even though it is not tailored for that task, achieving particularly good performance. Specifically, we explain how to build powerful dense SDMs that are suitable for objects that have rich interior texture but lack landmarks consistency. Furthermore, we show how to build a powerful patch-based SDM on the sparse outline landmarks of objects that do not have semantically meaningful interior textures. Using the resulting outline patch-based SDM, we report state-of-the-art performance on the task of human body parts localisation on challenging databases. Finally, we show that the proposed patch-based SDM can be used to provide consistent annotations for different body parts.

In summary, the contributions of this paper are:

- We propose one of the first, to the best of our knowledge, methodologies that constructs accurate SDMs from a set of training data with inconsistent annotations. We show that the proposed methodology tremendously reduces the manual workload thanks to the highly effective curve annotations.

- We illustrate the ability of the proposed method to generate consistent sparse landmark annotations for object classes which, by nature, make it impossible to be manually annotated in a consistent way.

- We show that it is more advantageous to model the human body parts (e.g. arms) with a set of sparse landmarks on their outline, rather than on their skeleton joints. This is because the outline landmarks, which can be acquired by our method in a straightforward way, exhibit better consistency compared to the inevitable inconsistency of the joint landmarks.

- We report state-of-the-art quantitative and qualitative results on human body parts localisation by employing a patch-based SDM trained on the outline landmarks that are sampled by the dense correspondences. Our proposed model outperforms all current state-of-theart techniques that are trained on skeleton joints.

- We show that the employed patch-based SDM corrects the annotations that are currently provided for most major human body pose databases ${ }^{2}$.

\footnotetext{
${ }^{2}$ The corrected annotations are publicly available in http://www.ibug.doc.ic.ac.uk/resources/ bodypose-anno-correction.
}

\section{Constructing Deformable Models with Shape Flow}

This section presents the proposed method for establishing dense correspondences among training shapes by only using curve annotations. It takes as input a set of training images of a particular object class, along with the corresponding curve annotations. The steps of our pipeline, which are also depicted in Figure 3, are the following:

Step 1: Represent the curve annotations in a consistent way using a multichannel extension of the Support Vector Shape (SVS) representation [44]. Apply the Iterative Closest Point (ICP) algorithm [16] to achieve an initial alignment of the SVS images.

Step 2: Construct a correspondence basis for the training SVS images. This is acquired by applying the Non-rigid ICP (NICP) algorithm of [5] on the densely sampled annotated curves, followed by Principal Component Analysis (PCA).

Step 3: Establish dense correspondences between all the shapes in the training set by feeding the multichannel similarity-aligned SVS images into a multi-image subspace flow estimation.

Step 4: Utilise the dense correspondences acquired by the optical flow in order to automatically generate either dense or sparse (on the outline) landmark annotations, depending on the object class type. Then, build either a dense $[48,4,6]$ or a patch-based [59] AAM, respectively.

The upcoming sections discuss each of the aforementioned steps in further detail.

\section{Step 1: Shape Representation Based on Support Vector Shapes}

In order to fully capture the variability among most deformable objects' shapes annotations, we use a representation based on SVS [44]. An SVS is a decision function trained on shapes using Support Vector Machines (SVMs) with Radial Basis Function (RBF) kernels. In this way, a shape is represented as a classifier function, which has several advantages: (a) the representation is completely generic, e.g. it can be applied to sparse landmark points, curves lines or a combination of the two, and (b) it fuses inconsistent landmarks into consistent and directly comparable decision functions. Furthermore, this representation is also robust against noise, missing data and outliers [44].

The curve annotations for all training images are densely sampled to yield a set of landmarks per image, with this set being different for every training image. To train the SVM, these landmarks are assigned as belonging to the 'positive' class, whereas randomly sampled points around them are assigned as belonging to the 'negative' class. Since the positive class has far less points than the negative class, landmarks are assigned considerably larger weights so that 


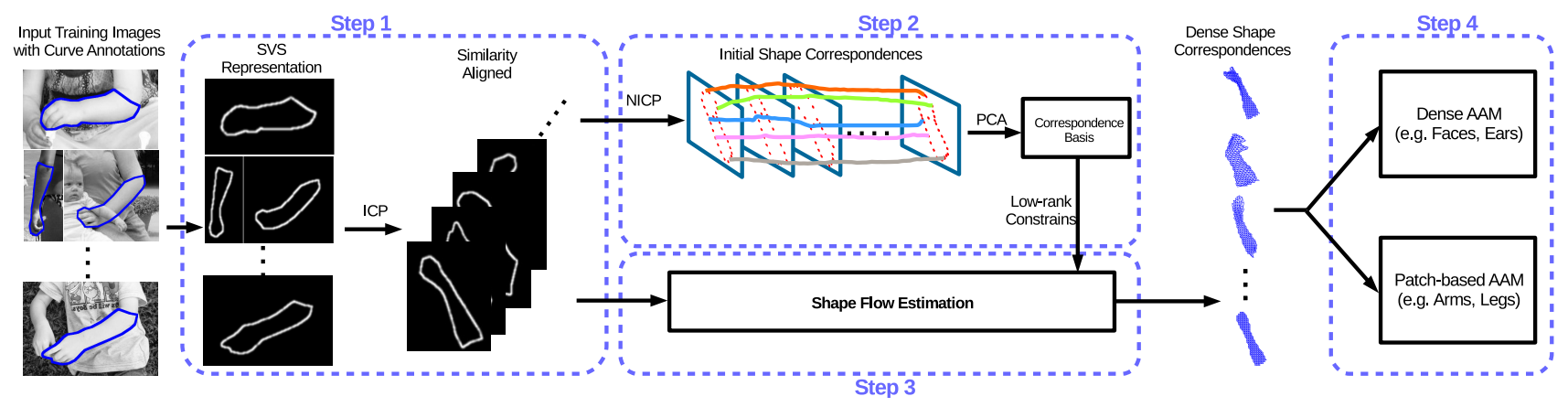

Figure 3: Schematic description of the proposed pipeline. Figure best viewed by zooming in.

$N_{p} \times W_{p}=N_{n} \times W_{n}$ where $N_{p}, N_{n}$ are number of points of the positive and negative class respectively and $W_{p}, W_{n}$ are their corresponding weights.

SVMs with RBF kernel functions map any number of data points onto an infinite-dimensional space where positive and negative points are linearly separable, hence the classification boundary on the $2 \mathrm{D}$ space represents the actual shape of the object. Let $d(\boldsymbol{x})$ be the decision function for SVMs. In our formulation, $d(\boldsymbol{x})$ can be defined for every pixel $\boldsymbol{x}$ of the corresponding object image, therefore we interpret it as an image and we call it SVS image.

We extend the SVS representation to support also the case where multiple curves with different labels are annotated. This is useful when annotating different structures of the same object, such as the left and right eyebrows on faces. Even though not absolutely necessary in our framework, it can provide further guidance on the estimation of dense shape correspondences for various object classes. In more detail, we create a multichannel SVS image $\boldsymbol{d}(\boldsymbol{x})=\left[d_{1}(\boldsymbol{x}) \cdots d_{i}(\boldsymbol{x}) \cdots d_{N_{c}}(\boldsymbol{x})\right]$, where $d_{i}(\boldsymbol{x})$ is the SVS image that corresponds to the curve annotation of the $i$-th structure and $N_{c}$ is the total number of structures (a single curve annotation is the special case where $N_{c}=1$ ). Note that we do not necessarily require that all structures are annotated in all the object images: in the case that a structure is not annotated, the corresponding channel of the SVS image simply takes a zero value for all pixels. The shape flow estimation can deal with such missing information thanks to the spatial regularization and the low-rank constraint that it adopts, c.f. Step 3.

After constructing the SVS representation for all images, the next step is to apply a simple similarity alignment over them. This is done because the goal here is to build a model capable of effectively representing non-rigid local shape deformations rather than global rotation, translation and scaling. The alignment is performed by using the ICP algorithm [16] on the annotated landmarks point cloud of the training images.

\section{Step 2: Correspondence Basis for Shape Flow Es- timation}

We define the problem of shape flow as the joint estimation of optical flow fields between a reference SVS image and every SVS image of the training dataset, which yields dense correspondences across SVS images. This also defines for every training SVS image a warping function that registers it with the reference SVS image. To establish the dense correspondences robustly, we are inspired by the idea of subspace constraints in the estimation of multiframe optical flow [31, 49, 55].

Instead of the motion basis used in multiframe optical flow formulation of [31], we build a correspondence basis that introduces constraints on how points of different shapes are matched to each other. Every pixel of the reference SVS image is matched to its corresponding position at every training SVS image and in this way defines a correspondence vector. This vector consists of the $2 \mathrm{D}$ locations of the specific point in all SVS images. To form this vector, the training images are arranged in an arbitrary order. Similarly to the order of the training samples when PCA is applied, this order does not affect the result of our method and any re-ordering would produce exactly the same results.

Formally, let $N_{t}$ be the number of training SVS images and $n=1, \ldots, N_{t}$ be the training image index. Also, let $\boldsymbol{q}_{1}(n), \ldots, \boldsymbol{q}_{R}(n):\left\{1, \ldots, N_{t}\right\} \rightarrow \mathbb{R}^{2}$ be the $R$ orthonormal elements of the correspondence basis, where $\boldsymbol{q}_{i}(n)$ is the displacement vector that matches the points of the reference SVS image with the points of the $n$-th training SVS image, according to the variation described from the $i$-th correspondence basis element. Note that the basis elements $\boldsymbol{q}_{i}(n)$ are independent from the point location. Note also that the number of basis elements is typically much smaller than the full dimensionality $\left(2 N_{t}\right)$ of correspondence vectors, therefore this basis plays a role of dimensionality reduction.

In addition, let $\Omega \subset \mathbb{R}^{2}$ be the image domain of the SVS images and $\boldsymbol{x}$ denote the point location. We denote the shape flow result as $\boldsymbol{u}_{n}(\boldsymbol{x}): \Omega \times\left\{1, \ldots, N_{t}\right\} \rightarrow \mathbb{R}^{2}$, where $\boldsymbol{u}_{n}(\boldsymbol{x})$ is the displacement vector that matches the 

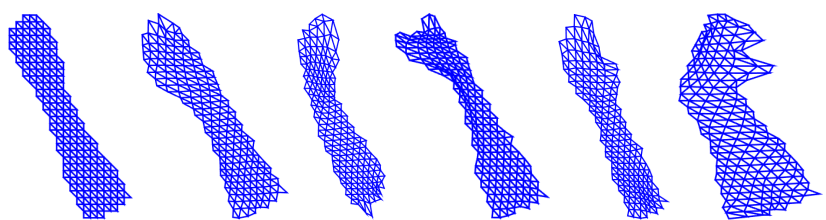

Figure 4: Exemplar deformation fields for the left arm, obtained using the proposed pipeline. Figure best viewed by zooming in.

point $\boldsymbol{x}$ of the reference SVS image with its corresponding location at the $n$-th training SVS image.

Using the constructed correspondence basis, the shape flow can be approximated as:

$$
\boldsymbol{u}_{n}(\boldsymbol{x}) \approx \sum_{i=1}^{R} \boldsymbol{q}_{i}(n) v_{i}(\boldsymbol{x})
$$

where $v_{i}(\boldsymbol{x})$ is the weight that needs to be applied on the $i$-th correspondence basis element, in order to get the correspondence vector for the point location $\boldsymbol{x}$. In other words, the shape flow for every point $\boldsymbol{x}$ is described as a linear combination of basis elements that is controlled by the coefficients $v_{i}(\boldsymbol{x})$. The values of the $i$-th coefficient for all the points $v_{i}(\boldsymbol{x})$ can be interpreted as an image defined on $\Omega$. Using the correspondence basis, the determination of the shape flow boils down to the determination of the set of coefficients $v_{i}(\boldsymbol{x})$. The above representation of shape flow, constrains the correspondence vectors to lie on a subspace and, therefore, acts as a low-rank prior that enforces coherency of the shape registration result over the whole training dataset of shapes.

To effectively build the correspondence basis, we first transform the original annotations to sparse point clouds. Then, we apply the NICP algorithm of [5] between the point cloud of annotations in the reference shape and the one of every shape of the training set. NICP iteratively deforms the cloud of points of every shape to match the points of the reference shape. This yields an initial estimation of the correspondence vectors on the sparse locations of annotated landmarks on the reference shape. Finally, the correspondence basis is found by applying PCA on these correspondence vectors and keeping only the first $R$ principal components.

\section{Step 3: Shape Flow Estimation}

As already mentioned, our shape flow estimation builds upon robust methods for multiframe optical flow estimation [31]. However, optical flow estimation typically works based on the assumptions of brightness or colour constancy and motion smoothness, whereas in our setting the input training data correspond to shapes. For this reason, we propose to modify the formulation of [31] by using the correspondence basis that we introduced in conjunction with the SVS representation of shapes.

Let $\boldsymbol{d}(\boldsymbol{x} ; n), \boldsymbol{d}(\boldsymbol{x} ; 0): \Omega \rightarrow \mathbb{R}^{N_{c}}$ be the $n$-th training SVS image and the reference SVS image respectively. Fol- lowing [31], we propose to estimate the shape flow over all training images by minimizing the following energy:

$$
\begin{aligned}
E_{s f} & =\alpha \int_{\Omega} \sum_{n=1}^{N_{t}}\left\|\boldsymbol{d}\left(\boldsymbol{x}+\boldsymbol{u}_{n}(x) ; n\right)-\boldsymbol{d}(x ; 0)\right\| \mathrm{d} \boldsymbol{x} \\
& +\beta \int_{\Omega} \sum_{n=1}^{N_{t}}\left\|\boldsymbol{u}_{n}(\boldsymbol{x})-\sum_{i=1}^{R} \boldsymbol{q}_{i}(n) v_{i}(\boldsymbol{x})\right\|^{2} \mathrm{~d} \boldsymbol{x} \\
& +\int_{\Omega} \sum_{i=1}^{R}\left\|\nabla v_{i}(\boldsymbol{x})\right\| \mathrm{d} \boldsymbol{x}
\end{aligned}
$$

This energy consists of two sets of unknown shape flows that are relatively close to each other: (i) $\boldsymbol{u}_{n}(x)$ which tries to explain the data from the input SVS images, and (ii) the shape flow determined by the correspondence basis coefficients $v_{i}(\boldsymbol{x})$ that are spatially regularised and enforce a low-rank prior.

The first term of the above energy (2) is a data attachment term that uses the robust $\mathbf{L}^{1}$-norm. It is based on the assumption that the values of the reference SVS image $\boldsymbol{d}_{0}(\boldsymbol{x})$ at every pixel $\boldsymbol{x}$ are preserved at its corresponding locations on all training SVS images $\boldsymbol{d}_{n}(\boldsymbol{x})$. The use of an $\mathbf{L}^{1}$-norm improves the robustness of the method since it allows deviations from this assumption, which might occur in practice. The second term of the energy (3) penalizes the difference between the two sets of shape flows and acts as a coupling term between them. The third term of the energy (4) corresponds to the spatial Total Variation regularization [50] of the correspondence basis coefficients $v_{i}(\boldsymbol{x})$. This term penalizes spatial oscillations of each coefficient caused by distortions of the SVS images but not strong discontinuities that are desirable in the borders of different object regions. In addition, this term allows to fill in information into regions where the shape information in the SVS images is missing, due to e.g. regions with no annotations.

We implement the minimization of the energy $E_{s f}$ by using the optimization algorithm described in [31]. For more details, please refer to the Supplementary Material. Figure 4 shows some examples of deformation fields derived from the estimated shape flow computed by the aforemen-

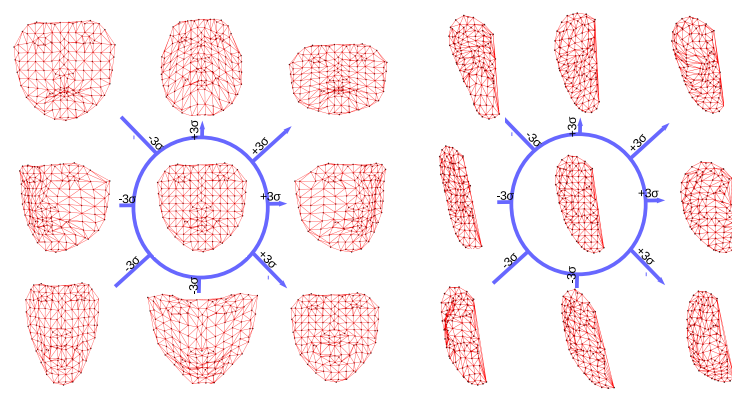

Figure 5: Dense shape models build for faces and ears. Dense shapes are presented as grid for better visualization. 
tioned method. These results correspond to exemplar training shapes in the case of an arm dataset. We observe that the shape flow estimation captures the shape and deformations of the human arm in a plausible way.

\section{Step 4: Dense and Patch-Based Deformable Models}

The deformation fields obtained from Step 3 can be used to naturally build two different kinds of effective Active Appearance Models (AAMs) [24, 43]: dense [48, 4, 6] and patch-based [59]. The only difference between these two AAM formulations is on the way that the shape is represented and, thus, the manner in which the texture is sampled. Each one of them is suitable for object classes with specific properties. The dense AAM provides an exceptionally effective modeling and fitting for non-articulated objects, such as ears and faces, whose appearance has characteristic structures that spread all over their region (even if these structures cannot be consistently annotated). On the other hand, there exist other challenging object classes, such as arms and legs, that not only cannot be consistently annotated with landmarks, but their appearance is distinctive only on the object's outline and not in its interior region. Especially in the case of human body parts, they are almost always covered by clothes, which makes it impossible to construct robust texture models.

Dense Active Appearance Model Since all the deformation fields acquired by Step 3 are defined for the pixels of the reference SVS image, the spatial positions $\boldsymbol{x}_{i}=\left(x_{i}, y_{i}\right)$ of these pixels can be treated as point landmarks and the deformation fields as dense annotations of the object's shape. Consequently, building a dense shape model reduces to normalising these dense annotations with respect to a global similarity transform (typically using Procrustes Analysis) and applying PCA. A shape instance can be generated by the resulting shape model as:

$$
s(\boldsymbol{p})=\bar{s}+\boldsymbol{S p}
$$

where $\overline{\boldsymbol{s}}$ is mean shape, and $\boldsymbol{S}$ and $\boldsymbol{p}$ are the shape bases and shape parameters, respectively.

By making explicit use of the one-to-one correspondence between pixels on the reference frame and on the deformation fields, the motion model of sparse holistic AAMs [24, 43] (piece-wise affine, thin-plates splines [17]) is replaced by sampling all pixel values onto the reference frame. Let us define this sampling function, given a shape instance $\boldsymbol{s}(\boldsymbol{p})$, as $\mathcal{W}(\boldsymbol{s}(\boldsymbol{p}))$. Once the images have been warped, the texture model is obtained by applying PCA on them. A texture instance can be generated as:

$$
t(c)=\bar{t}+T c
$$

where $t$ is the mean texture, and $\boldsymbol{T}$ and $\boldsymbol{c}$ are the texture bases and texture parameters, respectively.
Given a test image $\mathbf{I}$, the fitting process involves the minimization of the following cost function:

$$
\arg \min _{\boldsymbol{p}, \boldsymbol{c}}\|\boldsymbol{I}(\mathcal{W}(\boldsymbol{s}(\boldsymbol{p})))-\boldsymbol{t}(\boldsymbol{c})\|_{2}^{2}
$$

This optimization problem is typically solved using the inverse-compositional Gauss-Newton algorithm, for which different variations have been proposed [43, 45, 4, 58, 2] Note that the existence of the sampling function $\mathcal{W}()$ instead of a non-linear warping function has the advantage that all existing efficient gradient descent algorithms become exact.

Outline Patch-Based Active Appearance Model (PAAM) The object classes for which the interior appearance does not have specific structure are modeled using patch-based AAMs [59] trained on a set of sparse landmarks. Especially for human body parts (arms, legs), we strongly believe that the points located to the outline of the object are more suitable compared to the internal ones that correspond to the skeleton joints, which are commonly used by current literature [19, 22, 46, 61].

The main differences between the patch-based and dense AAMs are that (a) the densified shape instances are subsampled to include only the outline points, and (b) the texture representation involves the sampling a neighbourhood around each point instead of a single pixel. Specifically, in order to build the outline sparse shape model, we simply select the outline points on the SVS reference frame. Then, by taking advantage of the dense correspondences obtained by Step 3, the shape model is trained in a similar way as in the dense case. Moreover, similar to the dense case, the texture model is built by sampling the image values from the sparse shape locations, i.e. $\mathcal{W}(\boldsymbol{s}(\boldsymbol{p}))$. However, contrary to dense AAMs, we sample a patch that is centred around each landmark point. These patches are then vectorised and concatenated in a single texture vector. Note that the optimization process remains exactly the same.

\section{Experimental Evaluation}

We evaluate the performance of the proposed methodology for the task of human body pose correspondence estimation, as well as non-rigid alignment "in-the-wild". For further experimental results, please refer to the supplementary material. Note that all steps of the proposed pipeline were implemented using the Menpo Project [1].

\subsection{Non-rigid Object Alignment In-the-Wild}

Herein, we compare the fitting accuracy of the dAAMs that are trained with our proposed framework with holistic sparse AAMs [24, 43, 8]. We consider two object classes that demonstrate rich texture: face and ear. 


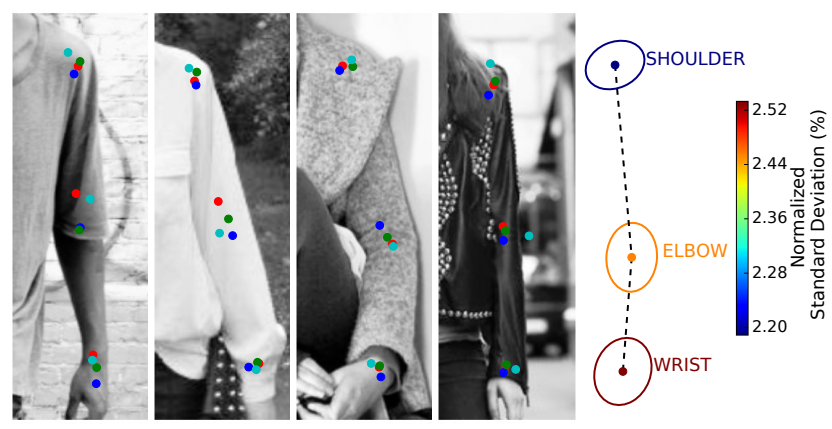

Figure 6: Example of human pose annotation for left arm among 4 annotators. The large variance highlights the difficulty of obtaining consistent landmarks.

Databases \& Error Metrics In the case of face, we trained both models using the 811 training images of the Labelled Faces Parts in-the-Wild (LFPW) [15]. Sparse AAMs were built from the 68 points annotations provided by $[52,51]$. Our dAAMs were built as described in Step 4. In both cases, the appearance is represented using pixel intensities. The results are reported on the 224 images of the LFPW testset. The fitting error is evaluated as the point-to-point distance normalised by the face's size, as proposed in [64].

In the case of human ear, given the lack of publicly available annotated databases, we collected 605 high resolution images captured under unconstrained conditions from online search engines. The images were manually annotated with respect to 55 sparse landmarks, as well as the curve annotations proposed in this paper. Examples of these two types of annotations are shown in Figure 2. We randomly split the database into two disjoint sets of training (500) and testing (105) images. The training and evaluation of the two models is done in the same way as in the case of face.

Results We report the results in Figure 9 using Cumulative Error Distribution (CED) curves. By visual inspection of the results, we determined that the fitting is adequately accurate for errors less than 0.1 and 0.06 for the ear and face, respectively. The results indicate that dAAMs marginally outperform sparse AAMs. Therefore, the proposed pipeline is capable of dealing with the complex structure of non-rigid shapes and train dAAMs from simple curve line annotations which can compete and even outperform the commonlyused sparse AAMs trained on carefully annotated images.

\subsection{Arm Pose Estimation}

In this experiment, we aim to compare the effect of training a deformable model of human arm using: (i) our proposed outline sparse landmarks, and (ii) the standard skeleton joints annotations that are commonly employed in literature. For this purpose, we employ the patch-based AAM as described in Step 4. Additionally, we compare our methodology with the current state-of-the-art.
Dataset \& Error Metric We opted to report quantitative results on the BBC Pose database [46], which provides the most consistent and accurate joints annotations compared to the rest of existing databases. The training of the outline patch-based AAM was performed after obtaining 29 outline landmarks using our proposed framework. We used 891 training images from a combination of datasets, including H3D [18], Microsoft COCO [38], MPII [7], Fashion Pose [28], FLIC [53] and BBC Pose [46]. SIFT features [18] are adopted for the image representation in our model. The fitting procedure on the BBC Pose database is initialised using a simplistic in-house deep convolutional neural network.

In order to compare with current state-of-the-art on BBC Pose, we used the same error metric as the one in [46], which normalises testing images in order to have a height of 256 pixels. Once again, the performance is visualised using CED curves. The results for this experiment are reported on 1000 testing images from BBC Pose, which utilises 7 skeleton landmarks to annotate the human upper-body pose. Note that in the case of our model, the final joints locations required for evaluation are retrieved from the dense correspondence acquired with our proposed method. On the contrary, the rest of the methods are trained on this 7-points mark-up, thus directly return their estimated locations.

Results Figure 10 reports the results of our model trained on the outline landmarks (Outline PAAM), as well as the current state-of-the-art techniques which include: Buehler [19], Charles14 [23], Charles13 [22], Pfister14 [47], Ramanan [61] and Pfister15 [46]. As can be seen, our outline part-based AAM model outperforms the state-of-the-art for this task, even though it is not trained directly on the wrist and elbow points, thus it is not tailored for locating them. In particular, our model outperforms the currently best method [46] by a notable amount (9\% with error less than 6pt) on wrist, as well as marginal improvement on elbow estimation. Figure 8 shows some indicative qualitative fitting results.

In the same experiment we prove that it is more advantageous to train a deformable model using the outline landmarks rather than the skeleton points. This is done by building a patch-based AAM on the same training data and with identical settings using both annotation schemes. As it can be seen from the CED curves of Figure 10, our model trained on outline landmarks (Outline PAAM) notable outperforms the skeleton-based model for both wrist and elbow. We believe that this is a remarkable result, which indicates that out proposed outline mark-up can lead to a significant improvement of current state-of-the-art techniques.

\subsection{Annotation Correction}

The final experiment demonstrates that it is feasible to use the proposed arm model in order to correct the anno- 

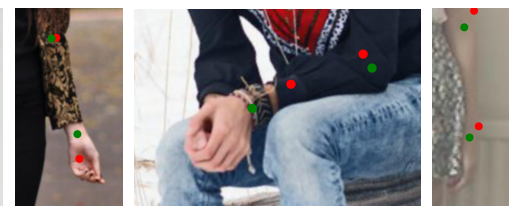

officially provided landmarks, and green dots are corrected position.
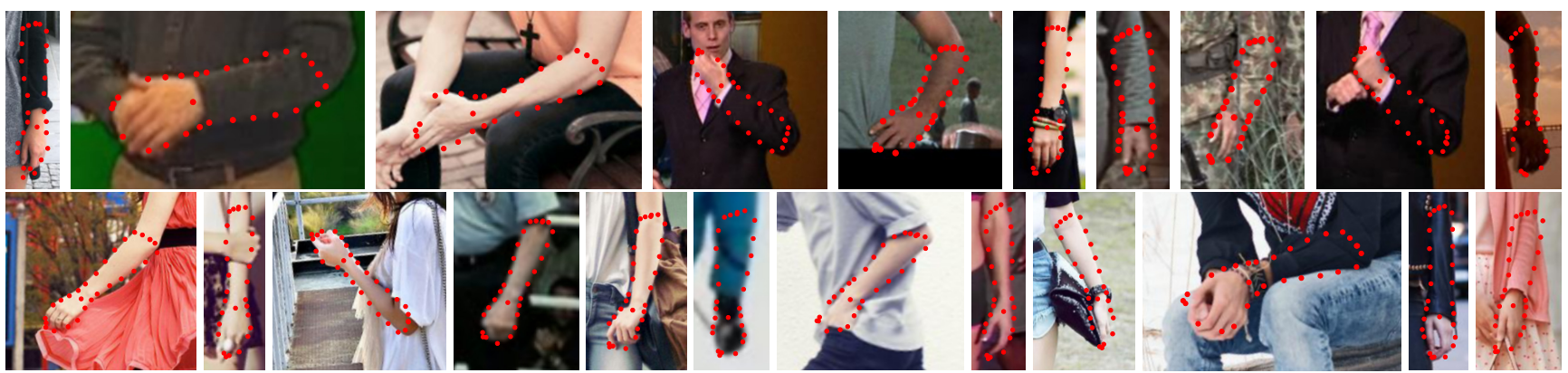

Figure 8: Demonstration of outline fitting of patch-based AAM on arms.

tations provided by current datasets. As mentioned above there are inconsistencies in the annotations of MPII [7], Fashion Pose [28] and FLIC [53]. Due to the large variance in arm pose, it is difficult even for trained annotators to obtain consistent annotations between them. As proof of concept, Figure 6 reports the standard deviation observed between the annotations of 4 trained humans that were requested to annotate 120 images of left arms from Fashion Pose [28] with respect to the shoulder, elbow and wrist.

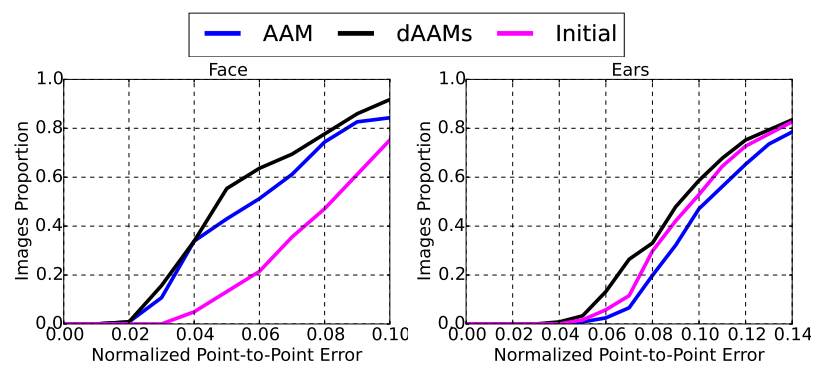

Figure 9: CEDs of faces and ears fitting performance for the experiment of Section 4.1.
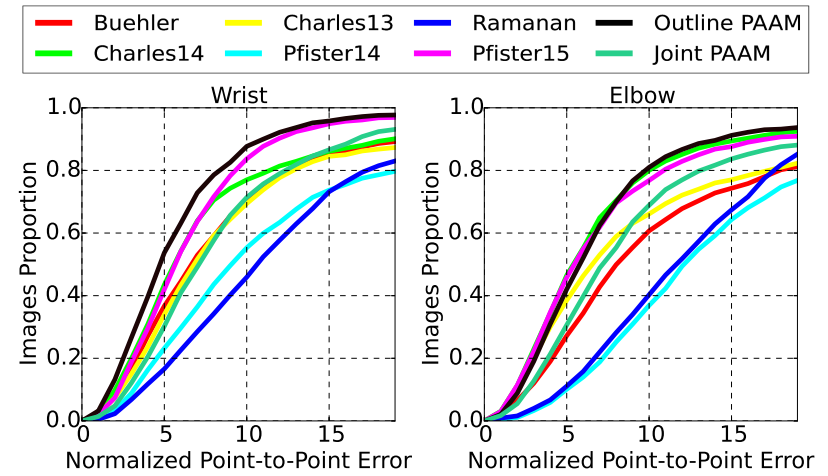

Figure 10: CEDs over skeleton landmarks on BBC Pose database for the experiment of Section 4.2.
By applying our outline patch-based AAM on the aforementioned databases, we managed to greatly correct the currently available annotations of the arm. Figure 7 shows indicative examples of the corrected landmarks. There is no doubt that points after correction demonstrate more consistency among images. We make the corrected annotations publicly available ${ }^{2}$.

\section{Conclusion}

Learning and fitting statistical deformable models (SDMs) is one of the most important areas in computer vision. Generally, in order to train a SDM, a set of predefined correspondences are required. In some objects, such as human face, semantically meaningful correspondences can be found, but require laborious manual annotations; on other objects it is very difficult, or even impossible. In this paper, we propose one of the first comprehensive procedures for establishing correspondences (that do not necessarily correspond to semantically meaningful object landmarks) in arbitrary objects with minimal amount of human annotation. We apply the proposed approach for the construction of the first, to the best of our knowledge, highly-descriptive SDM for the human arm.

Acknowledgements The work of E. Antonakos was partially funded by the EPSRC project EP/J017787/1 (4DFAB). The work of J. Alabort-i-Medina was partially funded by an EPSRC DTA. The work of A. Roussos was partially funded by the EPSRC project EP/N007743/1 (FACER2VM). The work of S. Zafeiriou was partially funded by the FiDiPro program of Tekes (project number: $1849 / 31 / 2015$ ), as well as by the European Community Horizon 2020 [H2020/2014-2020] under grant agreement no. 688520 (TeSLA). 


\section{References}

[1] J. Alabort-i-Medina, E. Antonakos, J. Booth, P. Snape, and S. Zafeiriou. Menpo: A comprehensive platform for parametric image alignment and visual deformable models. In Proceedings of the ACM International Conference on Multimedia, MM '14, pages 679-682, New York, NY, USA, 2014. ACM. 6

[2] J. Alabort-i-Medina and S. Zafeiriou. Bayesian active appearance models. In Conference on Computer Vision and Pattern Recognition (CVPR), 2014. 1, 6

[3] J. Alabort-i-Medina and S. Zafeiriou. Unifying holistic and parts-based deformable model fitting. In Proceedings of IEEE International Conference on Computer Vision and Pattern Recognition (CVPR'15), Boston, MA, USA, June 2015. 1

[4] B. Amberg, A. Blake, and T. Vetter. On compositional image alignment, with an application to active appearance models. In Conference on Computer Vision and Pattern Recognition (CVPR), 2009. 3, 6

[5] B. Amberg, S. Romdhani, and T. Vetter. Optimal step nonrigid ICP algorithms for surface registration. In Conference on Computer Vision and Pattern Recognition (CVPR), 2007. 3,5

[6] R. Anderson, B. Stenger, and R. Cipolla. Using bounded diameter minimum spanning trees to build dense active appearance models. International Journal of Computer Vision, 110(1):48-57, 2014. 3, 6

[7] M. Andriluka, L. Pishchulin, P. Gehler, and B. Schiele. 2d human pose estimation: New benchmark and state of the art analysis. In IEEE Conference on Computer Vision and Pattern Recognition (CVPR), June 2014. 2, 7, 8

[8] E. Antonakos, J. Alabort-i-Medina, G. Tzimiropoulos, and S. Zafeiriou. Feature-based lucas-kanade and active appearance models. IEEE Transactions on Image Processing, 24(9):2617-2632, September 2015. 1, 6

[9] E. Antonakos, J. Alabort-i-Medina, and S. Zafeiriou. Active pictorial structures. In Proceedings of IEEE International Conference on Computer Vision and Pattern Recognition (CVPR'15), pages 5435-5444, Boston, MA, USA, June 2015. 1

[10] E. Antonakos and S. Zafeiriou. Automatic construction of deformable models in-the-wild. In Proceedings of IEEE International Conference on Computer Vision \& Pattern Recognition (CVPR'14), pages 1813-1820, Columbus, $\mathrm{OH}$, USA, June 2014. 2

[11] A. Asthana, S. Zafeiriou, S. Cheng, and M. Pantic. Robust discriminative response map fitting with constrained local models. In Conference on Computer Vision and Pattern Recognition (CVPR), 2013. 1

[12] A. Asthana, S. Zafeiriou, S. Cheng, and M. Pantic. Incremental face alignment in the wild. In Conference on Computer Vision and Pattern Recognition (CVPR), 2014. 1, 2

[13] S. Baker, I. Matthews, and J. Schneider. Automatic construction of active appearance models as an image coding problem. IEEE Transactions on Pattern Analysis and Machine Intelligence, 26(10):1380, 2004. 2
[14] P. N. Belhumeur, D. W. Jacobs, D. J. Kriegman, and N. Kumar. Localizing parts of faces using a consensus of exemplars. In Conference on Computer Vision and Pattern Recognition (CVPR), 2011. 1

[15] P. N. Belhumeur, D. W. Jacobs, D. J. Kriegman, and N. Kumar. Localizing parts of faces using a consensus of exemplars. Pattern Analysis and Machine Intelligence, IEEE Transactions on, 35(12):2930-2940, 2013. 7

[16] P. Besl and N. D. McKay. A method for registration of 3-d shapes. Transactions on Pattern Analysis and Machine Intelligence (TPAMI), 1992. 3, 4

[17] F. J. Bookstein. Principal warps: Thin-plate splines and the decomposition of deformations. Transactions on Pattern Analysis and Machine Intelligence (TPAMI), 11(6):567-585, 1989. 6

[18] L. Bourdev and J. Malik. Poselets: Body part detectors trained using $3 \mathrm{~d}$ human pose annotations. In International Conference on Computer Vision, sep 2009. 7

[19] P. Buehler, M. Everingham, D. P. Huttenlocher, and A. Zisserman. Upper body detection and tracking in extended signing sequences. International journal of computer vision, 95(2):180-197, 2011. 6, 7

[20] X. P. Burgos-Artizzu, P. Perona, and P. Dollr. Robust face landmark estimation under occlusion. In International Conference on Computer Vision (ICCV), 2013. 1

[21] X. Cao, Y. Wei, F. Wen, and J. Sun. Face alignment by explicit shape regression. In Computer Vision and Pattern Recognition (CVPR), 2012. 1, 2

[22] J. Charles, T. Pfister, D. Magee, D. Hogg, and A. Zisserman. Domain adaptation for upper body pose tracking in signed tv broadcasts. In Proceedings of the British machine vision conference, 2013. 2, 6, 7

[23] J. Charles, T. Pfister, D. Magee, D. Hogg, and A. Zisserman. Upper body pose estimation with temporal sequential forests. In Proceedings of the British Machine Vision Conference 2014, pages 1-12. BMVA Press, 2014. 7

[24] T. F. Cootes, G. J. Edwards, and C. J. Taylor. Active appearance models. Transactions on Pattern Analysis and Machine Intelligence (TPAMI), 2001. 1, 6

[25] T. F. Cootes, S. Marsland, C. J. Twining, K. Smith, and C. J. Taylor. Groupwise diffeomorphic non-rigid registration for automatic model building. In Computer Vision-ECCV 2004, pages 316-327. Springer, 2004. 2

[26] T. F. Cootes, C. J. Taylor, D. H. Cooper, and J. Graham. Active shape models: Their training and application. Computer Vision and Image Understanding, 1995. 1

[27] N. Dalal and B. Triggs. Histograms of oriented gradients for human detection. In Conference on Computer Vision and Pattern Recognition (CVPR), pages 886-893, 2005. 2

[28] M. Dantone, J. Gall, C. Leistner, and L. Van Gool. Human pose estimation using body parts dependent joint regressors. In Computer Vision and Pattern Recognition (CVPR), 2013 IEEE Conference on, pages 3041-3048. IEEE, 2013. 7, 8

[29] M. Dantone, J. Gall, C. Leistner, and L. Van Gool. Body parts dependent joint regressors for human pose estimation in still images. Pattern Analysis and Machine Intelligence, IEEE Transactions on, 36(11):2131-2143, 2014. 2 
[30] B. J. Frey, M. Jojic, and A. Kannan. Learning appearance and transparency manifolds of occluded objects in layers. In Computer Vision and Pattern Recognition, 2003. Proceedings. 2003 IEEE Computer Society Conference on, volume 1, pages I-45. IEEE, 2003. 2

[31] R. Garg, A. Roussos, and L. Agapito. A variational approach to video registration with subspace constraints. International Journal of Computer Vision, 104(3):286-314, 2013. 2, 3, 4, 5

[32] X. Huang, N. Paragios, and D. Metaxas. Shape registration in implicit spaces using information theory and free form deformations. Pattern Analysis and Machine Intelligence, IEEE Transactions on, 28(8):1303-1318, Aug 2006. 2

[33] T. Jiang, F. Jurie, and C. Schmid. Learning shape prior models for object matching. In Computer Vision and Pattern Recognition, 2009. CVPR 2009. IEEE Conference on, pages 848-855. IEEE, 2009. 2

[34] N. Jojic, J. Winn, and L. Zitnick. Escaping local minima through hierarchical model selection: Automatic object discovery, segmentation, and tracking in video. In Computer Vision and Pattern Recognition, 2006 IEEE Computer Society Conference on, volume 1, pages 117-124. IEEE, 2006. 2

[35] V. Kazemi and J. Sullivan. One millisecond face alignment with an ensemble of regression trees. In Proceedings of the IEEE Conference on Computer Vision and Pattern Recognition, pages 1867-1874, 2014. 1

[36] I. Kokkinos and A. Yuille. Unsupervised learning of object deformation models. In Computer Vision, 2007. ICCV 2007. IEEE 11th International Conference on, pages 1-8. IEEE, 2007. 2

[37] V. Le, B. Jonathan, Z. Lin, L. Boudev, and T. S. Huang. Interactive facial feature localization. In European Conference on Computer Vision (ECCV), 2012. 1

[38] T.-Y. Lin, M. Maire, S. Belongie, J. Hays, P. Perona, D. Ramanan, P. Dollár, and C. L. Zitnick. Microsoft coco: Common objects in context. In Computer Vision-ECCV 2014, pages 740-755. Springer, 2014. 7

[39] S. Liu, X. Liang, L. Liu, X. Shen, J. Yang, C. Xu, L. Lin, X. Cao, and S. Yan. Matching-cnn meets knn: Quasi-parametric human parsing. arXiv preprint arXiv:1504.01220, 2015. 2

[40] X. Liu, Y. Tong, and F. W. Wheeler. Simultaneous alignment and clustering for an image ensemble. In Computer Vision, 2009 IEEE 12th International Conference on, pages 13271334. IEEE, 2009. 2

[41] D. G. Lowe. Object recognition from local scale-invariant features. In Computer vision, 1999. The proceedings of the seventh IEEE international conference on, volume 2, pages 1150-1157. Ieee, 1999. 2

[42] P. Luo, X. Wang, and X. Tang. Pedestrian parsing via deep decompositional network. In Computer Vision (ICCV), 2013 IEEE International Conference on, pages 2648-2655. IEEE, 2013. 2

[43] I. Matthews and S. Baker. Active appearance models revisited. International Journal of Computer Vision (IJCV), 2004. 1,6
[44] H. V. Nguyen and F. Porikli. Support vector shape: A classifier-based shape representation. Transactions on Pattern Analysis and Machine Intelligence (TPAMI), 2013. 2, 3

[45] G. Papandreou and P. Maragos. Adaptive and constrained algorithms for inverse compositional active appearance model fitting. In Conference on Computer Vision and Pattern Recognition (CVPR), 2008. 6

[46] T. Pfister, J. Charles, and A. Zisserman. Flowing convnets for human pose estimation in videos. arXiv preprint arXiv:1506.02897, 2015. 6, 7

[47] T. Pfister, K. Simonyan, J. Charles, and A. Zisserman. Deep convolutional neural networks for efficient pose estimation in gesture videos. In Computer Vision-ACCV 2014, pages 538-552. Springer, 2015. 7

[48] K. Ramnath, S. Baker, I. Matthews, and D. Raman. Increasing the density of active appearance models. In Computer Vision and Pattern Recognition, 2008. CVPR 2008. IEEE Conference on, pages 1-8. IEEE, 2008. 3, 6

[49] S. Ricco and C. Tomasi. Dense lagrangian motion estimation with occlusions. In CVPR, pages 1800-1807, 2012. 2, 4

[50] L. Rudin, S. Osher, and E. Fatemi. Nonlinear total variation based noise removal algorithms. Physica D, 60:259-268, 1992. 5

[51] C. Sagonas, E. Antonakos, G. Tzimiropoulos, S. Zafeiriou, and M. Pantic. 300 faces in-the-wild challenge: Database and results. Image and Vision Computing, Special Issue on Facial Landmark Localisation "In-The-Wild”, 2016. 2, 7

[52] C. Sagonas, G. Tzimiropoulos, S. Zafeiriou, and M. Pantic. 300 faces in-the-wild challenge: The first facial landmark localization challenge. In Proceedings of IEEE International Conference on Computer Vision (ICCV-W 2013), 300 Faces in-the-Wild Challenge (300-W), Sydney, Australia, December 2013. 2, 7

[53] B. Sapp and B. Taskar. Modec: Multimodal decomposable models for human pose estimation. In Computer Vision and Pattern Recognition (CVPR), 2013 IEEE Conference on, pages 3674-3681. IEEE, 2013. 7, 8

[54] J. M. Saragih, S. Lucey, and J. F. Cohn. Deformable model fitting by regularized landmark mean-shift. International Journal of Computer Vision (IJCV), 2011. 1

[55] P. Snape, A. Roussos, Y. Panagakis, and S. Zafeiriou. Face flow. In International Conference on Computer Vision (ICCV), pages 2993-3001. IEEE, 2015. 2, 4

[56] J. Tompson, R. Goroshin, A. Jain, Y. LeCun, and C. Bregler. Efficient object localization using convolutional networks. In Proceedings of the IEEE Conference on Computer Vision and Pattern Recognition, pages 648-656, 2015. 2

[57] G. Tzimiropoulos. Project-out cascaded regression with an application to face alignment. In Proceedings of IEEE International Conference on Computer Vision and Pattern Recognition (CVPR'15), pages 3659-3667. IEEE, 2015. 1

[58] G. Tzimiropoulos and M. Pantic. Optimization problems for fast aam fitting in-the-wild. In International Conference on Computer Vision (ICCV), 2013. 6

[59] G. Tzimiropoulos and M. Pantic. Gauss-newton deformable part models for face alignment in-the-wild. In Conference on 
Computer Vision and Pattern Recognition (CVPR), 2014. 1, 2, 3,6

[60] Xuehan-Xiong and F. De la Torre. Supervised descent method and its application to face alignment. In Conference on Computer Vision and Pattern Recognition (CVPR), 2013. 1,2

[61] Y. Yang and D. Ramanan. Articulated human detection with flexible mixtures of parts. Pattern Analysis and Machine Intelligence, IEEE Transactions on, 35(12):2878-2890, 2013. 6,7

[62] P. Zhang and T. Cootes. Automatic construction of parts+geometry models for initializing groupwise registration. Medical Imaging, IEEE Transactions on, 31(2):341358, Feb 2012. 2

[63] S. Zhu, C. Li, C. Change Loy, and X. Tang. Face alignment by coarse-to-fine shape searching. In Proceedings of the IEEE Conference on Computer Vision and Pattern Recognition, pages 4998-5006, 2015. 1

[64] X. Zhu and D. Ramanan. Face detection, pose estimation, and landmark localization in the wild. In Conference on Computer Vision and Pattern Recognition (CVPR), 2012. 1, 2,7

[65] S. Zuffi, O. Freifeld, and M. J. Black. From pictorial structures to deformable structures. In Computer Vision and Pattern Recognition (CVPR), 2012 IEEE Conference on, pages 3546-3553. IEEE, 2012. 2 